\title{
Three-dimensional Face Reconstruction using 3D Morphable Model Fitting Method
}

\author{
Shaimaa H. Shaker \\ University of Technology \\ Department of Computer Sciences \\ sh_n_s2004@yahoo.com
}

\author{
Najlaa Abd Hamza \\ University of Baghdad \\ College of Nursing \\ najlaa_al_mayyahee@yahoo.com
}

Recived : $30 \backslash 5 \backslash 2018$

Revised : $31 \backslash 5 \backslash 2018$

Accepted : 10\6\2018

Available online : $\quad$ 8/8/2018

DOI: $10.29304 / j q c m .2018 .10 .3 .418$

\begin{abstract}
In this paper, the reconstruction of faces in the form of a 3D model from unconstrained images (different in pose) has been studied. Three-dimensional Morphable Model(3DMM) Fitting method has been used in 3D reconstruction techniques. In this paper propose improvement of the 3DMM fitting procedure to get accurate 3D model by taking best 2D landmark of all images rather than taking only first 2D image landmark (traditional method which proposed by A.Bas et al). The results of the proposed algorithm show its very encouraging as far as execution time and quality of reconstruction as shown in compare the improvement fitting process with the traditional method.
\end{abstract}




\section{Introduction}

Face reconstruction is the way toward making a 3D model of a face from twoDimension (2D) image(s) [1][2].

It is important to consider in mind that creation human face models should look like as genuine image as possible. This procedure involves a transformation from $2 \mathrm{D}$ to $3 \mathrm{D}$ spaces[3].

Face reconstruction is a significant application in face recognition, video editing, virtual reality, animation [4], verification, expression recognition and facial animations [5].
For example, exact face models have been appeared to fundamentally progress face recognition [4]. In every one of these applications, the reconstructed face should be compacted and precised, particularly around, and so on. Computer games can be appeared as a good illustration which needs accurate human face models [3]. Fig.1 shows general points identified with the execution of 3D face reconstruction procedures. Most of the 3D reconstruction algorithms share the same basic processing pipeline, and may be not running all the processing steps [4].

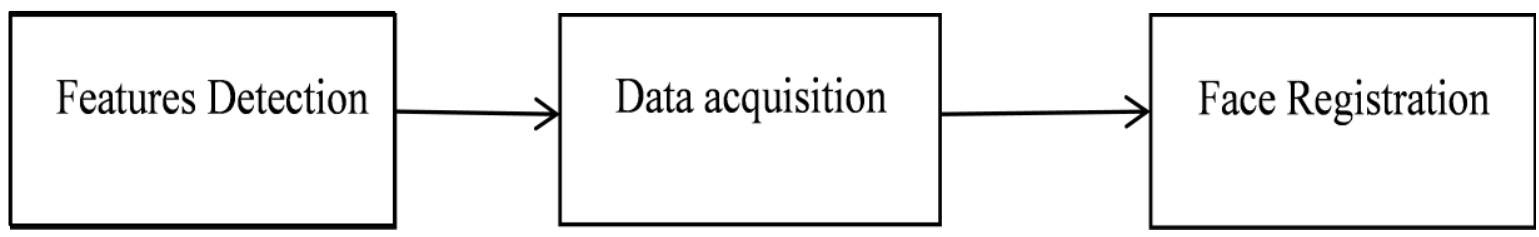

Figure 1: General steps of 3D reconstruction.

The determination of important features in the input 2D image(s) is a significant stage in most 3D face reconstruction procedures. Usually face feature determination has been utilized for [1][6]:

1. Introducing the situating of face models in $3 \mathrm{D}$ reconstruction methods based on the model.

2. Finding feature on sideways and forward images so it is conceivable to misshape general 3D face prototypes to accept the form of the specified face.

3. Instating the procedure of point tracing in $3 \mathrm{D}$ reconstruction methods based on video.

4. Creating point consistency in faces taken from unlike vantage point

Another important part of the reconstruction process is "data acquisition". It is gathering

the $3 \mathrm{D}$ information about that object by using one of $3 \mathrm{D}$ reconstruction technique. In this paper 3D MM model fitting procedure has been used in reconstruction of 3D faces.
A significant task of $3 \mathrm{D}$ reconstruction methods is the face registration. It is bringing the whole generic 3D model vertices as near as could be expected under the corresponding to the relating $3 \mathrm{D}$ coordinates of the feature points which computed from images. The opposite is also potential, i.e., transporting the intended 3D points near to the generic 3D model. This corresponding involves scaling, translating and rotating of the one to be moved closer to the other.

The 3D registration approaches can be classified into two distinct collections [4]:

1. Three-dimensional to $3 D$ registration: Corresponding is done among two 3D points. One is a base mesh, and other one is the $3 \mathrm{D}$ data produced in anyway after the data acquisition procedure. 


\section{Three-dimensional to $2 \mathrm{D}$ registration:}

These methods achieve a 3D model which is utilized to fit $2 \mathrm{D}$ data.

In this work, registration approach is applied to perform model fitting. The goal of this paper is reconstruction multiple unconstrained image (different in pose) in the form of 3D model. In the rest of this paper, show a 3D Model Fitting approach in section 2, display a 3D Morphable Model and Fitting with 2D Landmarks and edges in Section 3, section 4 displays the proposed 3D Face Reconstruction, section 5 displays result and Discussion, section 6 shows the conclusions and, finally, a list of the references in section 7

\section{Three-dimensional Model Fitting approach}

The fitting is actually an optimization process, targeting to discovery the parameters of best model usually by minimizing the difference among the input images and model reconstructed/synthesized image[7]. The 3D model fitting can reconstruct the camera model and 3D shape, lighting texture, from a single image as shown in Fig. 2 [8]. The recovered parameters can then be used for face reconstruction.

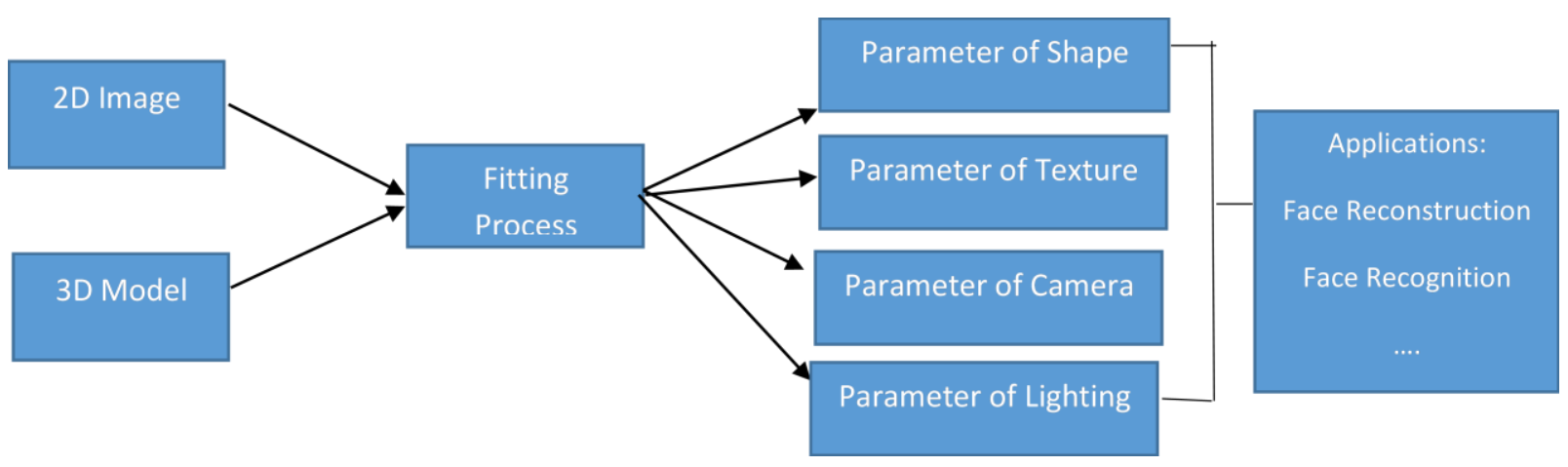

Figure 2: 3D model fitting.

In the proposed system, perform fitting process of a 3D model to a 2D images according to features (landmarks and edges). In the following sections, a detailed introduction of the model fitting approach will be given.

\section{Three-dimensional}

\section{Model}

The Morphable Model is the one applied by The University of Basel in 2009. Its name is Basel Face Model [9]. It's a mesh can be deform where shape is specified by the shape parameters $\alpha \in \mathrm{R}^{S}$. Consequently, any face shape can be estimated as[10]:

$$
f(\alpha)=C \alpha+\bar{m}
$$


where $\mathrm{C} \in \mathrm{N}^{3 \mathrm{R} \times \mathrm{S}}$ holds the principal components, $\bar{m} \in N^{3 R}$, is the shape of mean and the vector $\mathrm{f}$ $(\alpha) \in \mathrm{N}^{3 \mathrm{R}}$ holds the vertices coordinates $(\mathrm{R})$, accumulated to compose a vector $f=$ $\left[\begin{array}{llllll}x_{1} & y_{1} & z_{1} & \ldots & x_{R} & y_{R} z_{R}\end{array}\right]^{T}$. Later, the $j$ th vertex is specified by $U_{j}=\left[\begin{array}{lll}f_{3 j-2} & f_{3 j-1} & f_{3 j}\end{array}\right]^{T}$. For suitability, indicate the submatrix matching to the $\mathrm{j}$ th vertex as $C_{j} \in N^{3 \times S}$ and the matching vertex in the shape of mean face as $\overline{m_{j}} \in \mathrm{N}^{3}$, such that the $j$ th vertex is given by $U_{j}=C_{j \alpha}+\overline{m_{\imath}} \quad$ also, describes the row equivalent to the element ( $\mathrm{x}$ ) of the $\mathrm{jth}$ vertex as $\mathrm{C}_{\mathrm{jx}}$ (correspondingly for $\mathrm{y}$ and $\mathrm{z}$ ) and describe the element (y) of the jth vertex of mean shape as $\overline{m_{J x}}$ (correspondingly for $\mathrm{z}$ and $\mathrm{x})[7][11]$.

\subsection{Fitting with 2D Landmarks}

Fitting of 3DMM to $\mathrm{N}$ detected 2D locations(landmarks) $w_{j}=\left[\begin{array}{ll}a_{j} & b_{j}\end{array}\right]^{T}(j=1 \ldots N)$ rising on the projection of matching vertices in the 3DMM. Without wastage of generalization, suppose that the $j$ th $2 \mathrm{D}$ location matches to the $j$ th vertex in the 3DMM. The goal of fitting process is to get the parameters of pose and shape that minimize the reprojection error, $\mathrm{E}_{\mathrm{k}}$, among experiential and projected 2D locations:

$E_{k}(\alpha, O, r, l)=\frac{1}{N} \sum_{j=1}^{N}\left\|x_{j}-\left[C_{j} \alpha+\overline{m_{J}}, R, t, s\right]\right\|^{2}$

Where $\mathrm{O}$ is rotation matrix, $\mathrm{r}$ is translation vector and $l$ represents Scale.

Pose Estimation represents extraction of $O, r$ and $l$. let double duplicates of the points (3D), such

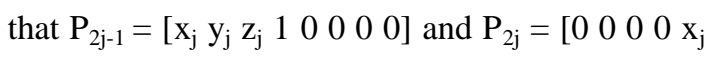
$\left.\begin{array}{llll}y_{j} & z_{j} & 1\end{array}\right]$ and compose a vector of the equivalent points(2D) $\mathrm{W}=\left[\begin{array}{lllll}a_{1} & b_{1} & \ldots & a_{L} & b_{L}\end{array}\right]^{T}$. subsequently solve for $\mathrm{d} \in \mathrm{R}^{8}$ in $\mathrm{Pd}=\mathrm{W}$ utilize linear least squares. describe $\mathrm{o}_{1}=\left[\begin{array}{lll}\mathrm{d}_{1} & \mathrm{~d}_{2} & \mathrm{~d}_{3}\end{array}\right]$ and $\mathrm{o}_{2}=\left[\begin{array}{ll}\mathrm{d}_{5} & \mathrm{~d}_{6}\end{array}\right.$ $\mathrm{d}_{7}$ ]. Scale is specified by $l=\left(\left\|o_{1}\right\|+\left\|o_{2}\right\|\right) / 2$ and the vector of translation by $\mathrm{r}=\left[\begin{array}{lll}\mathrm{d}_{4} / l & \mathrm{~d}_{8} / & l\end{array}\right]^{\mathrm{T}}$. achieve SVD on the matrix established from $\mathrm{o}_{1}$ and $\mathrm{o}_{2}[12]$ :

$$
U S V^{T}=\left[\begin{array}{c}
o_{1} \\
o_{1} \\
o_{1} \times o_{1}
\end{array}\right]
$$

The matrix of rotation is specified by $O=U V^{T}$. If $\operatorname{det}(O)=-1$ then refuse the third row of $(\mathrm{U})$ and recomput $(\mathrm{O})$. This assurance that $(\mathrm{O})$ is a matrix of valid rotation.

The 2D location of the $j$ th vertex as a function of the parameters of shape is assumed by $l \mathrm{O}_{1 . .2}\left(\mathrm{C}_{\mathrm{j} \alpha}+\overline{m_{\jmath}}\right)+l$ r. Later, each detected vertex complements (two) equations of a linear system, for both image compose the matrix $P \in R^{2 L \times S}$ wherever

$P_{2 j-1}=l\left(O_{11} C_{j x}^{T}+O_{12} C_{j y}^{T}+O_{13} C_{j z}^{T}\right)$

and

$$
P_{2 i}=l\left(O_{21} C_{j x}^{T}+O_{22} C_{j y}^{T}+O_{23} C_{j z}^{T}\right)
$$

and vector $\mathrm{k} \in \mathrm{R}^{2 \mathrm{~L}}$ where

$k_{2 j-1}=a_{i}-l\left(O_{1} \bar{m}_{j}+r_{1}\right) \quad$ and $\quad k_{2 j}=b_{j}-$ $l\left(\mathrm{O}_{2} \bar{m}_{j}+r_{2}\right)$

solve $P \alpha=k$ in a logic of least squares topic to an extra limitation to guarantee believability of the explanation[12].

\subsection{Iterated Closest Edge Fitting}

It's a method to fit a $3 \mathrm{DMM}$ to $2 \mathrm{D}$ edges. That is, for both vertex of projected model contour, discovery the nearby pixel of image edge and handle this as an identified matching. In combination with the landmark matching, will again perform the process in sections 3.1. 
This leads to updated parameters of shape and pose, trying to update edges of model and correspondences.Repeat this procedure for a static amount of repetitions. Denote to this procedure as (ICEF) Iterated Closest Edge Fitting. Discover the pixel of image edge nearby to a vertex of projected contour can be completed powerfully by storage the pixels of image edge in a kd-tree. Then filter the resulting matching using two generally utilized heuristics. Initialy, eliminate $5 \%$ of the correspond for which the space to the pixel of neighboring image edge is the biggest. Subsequent, eliminate correspond image space divided by $n$ overtake a threshold.

\section{The Proposed 3D Face}

\section{Reconstruction System}

The Proposed 3D face reconstruction system begins from obtaining "unconstrained" collection of face images captured under a varied of poses. the ending of proposed procedure is deriving a 3D face model with texture information. The workflow of the proposed approach could be outlined as follows and as shown in figure 3:
- Read 3D general Model, in this paper '.ply' file format are used (Morphable model). Algorithm (1) contains the main steps needed to read 3D model file.

- Load parameters of initial models. The parameters are:

1. verticesPC is a $3 n$ by $k$ matrix where $\mathrm{n}$ is the model vertices number and $\mathrm{k}$ the principal components number.

2. verticesMU is a $3 n$ by 1 vector containing the vertices of the mean shape.

3. StandardPC is a $\mathrm{k}$ by 1 vector containing the sorted standard deviations of each principal component.

$\mathrm{fl}$ is an $\mathrm{n}$ by 3 matrix containing the face list for the model. 

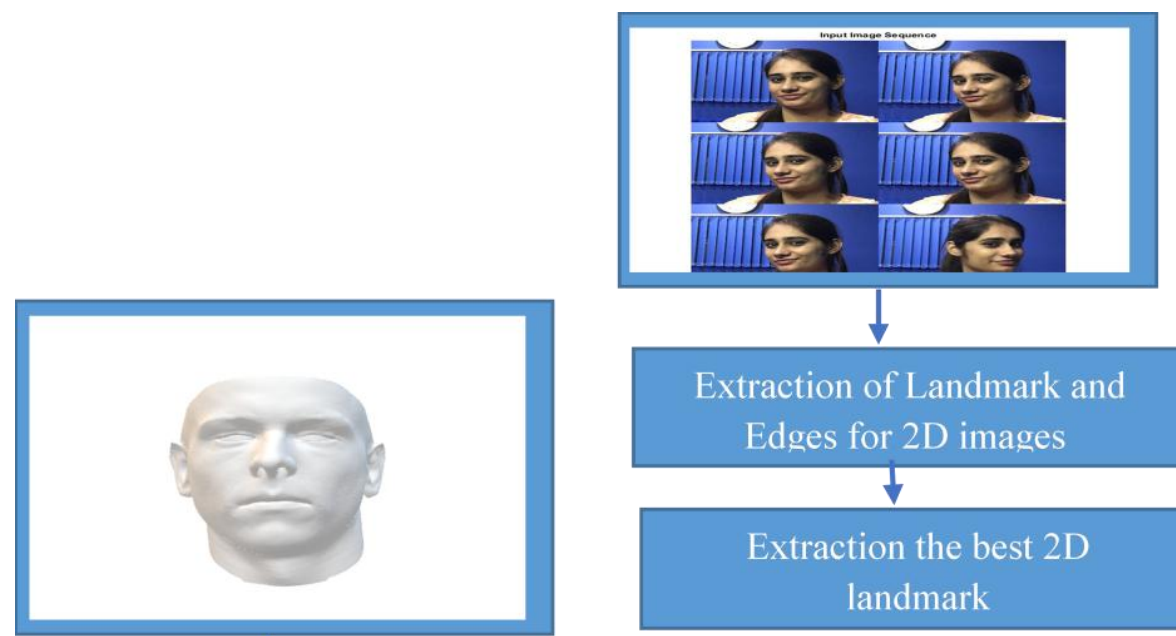

Extraction of Landmark and Edges for 2D images

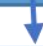

Extraction the best 2D

landmark

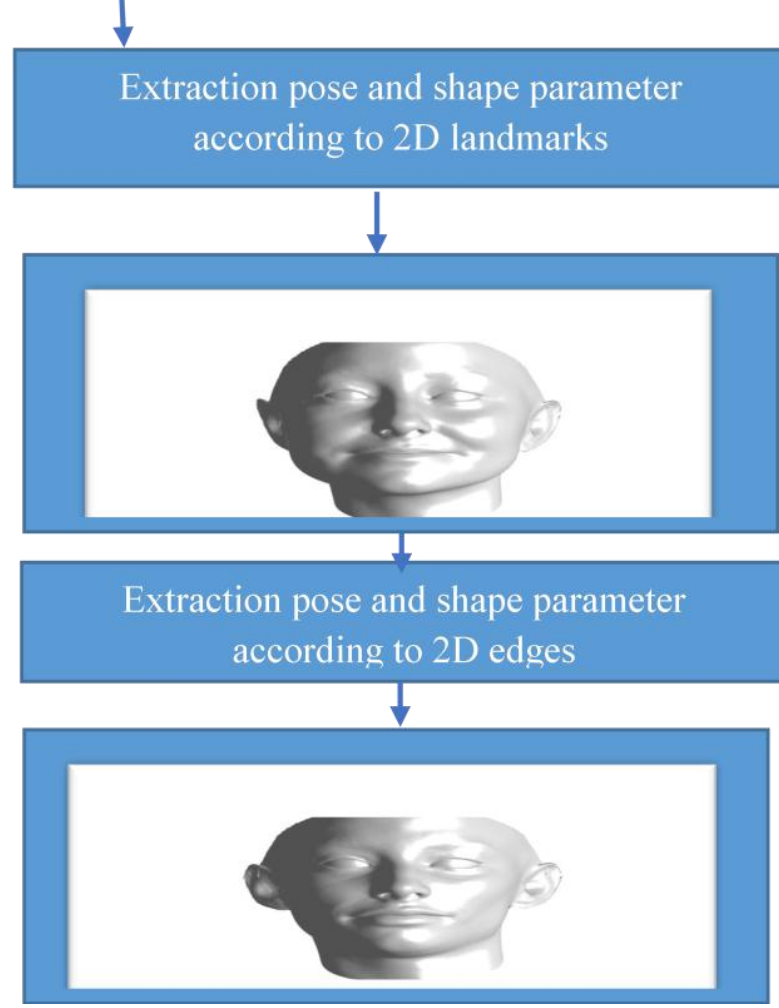

Figure3: The Block Diagram of the improvement 3D MM model fitting Method 


\section{Algorithm(1): Read of 3D Model}

Input: 3D file

Output: Faces array (F), Vertices $\operatorname{array}(\mathrm{V})$

\section{Begin}

//Read the size of vertices from 3D file

Vertices no. $=$ size $(\mathrm{V})$

For $\mathrm{i}=1$ : Vertices no // read the vertices of $3 \mathrm{D}$ model

Read (Vi)

End

// Read the size of faces from 3D file

Faces No $=\operatorname{size}(\mathrm{F})$

For $\mathrm{i}=1$ : faces no. // read the faces of $3 \mathrm{D}$ model

Read (Fi)

End

End

- Load precomputed edge structure for initial models.

- Read sequence of images (2D image).

- Find edges in this 2D image by using Canny methods. The Canny technique discoveries edges by searching for local greatest of the image gradient. The gradient is calculated utilize the derivative of a filter of Gaussian. The method uses two thresholds, to detect strong and weak edges, and includes the weak edges in the output only if they are connected to strong edges. This method is therefore more than the others methods to be "robust" by noise, and more likely to detect true weak edges

- Extraction the landmark of the 2D image by using the method which proposed by [13].
- Initial estimate of pose and shape parameters of finial 3D face model using only location of 2D landmarks as shown in subsection 3.1. In this step, proposed improvement of fitting process to get accurate landmark by taking best 2D landmark of all images rather than taking only first $2 \mathrm{D}$ image landmark (traditional method). the step of modifying detection of best landmark shown in the following algorithm (2), while the step of extraction of pose and shape parameters according to 2D landmark shown in the algorithm (3) 


\section{Algorithm(2): Detection best of 2D landmark}

Input: serial of 2D image, 2D landmark

Output: detection of best 2D landmark

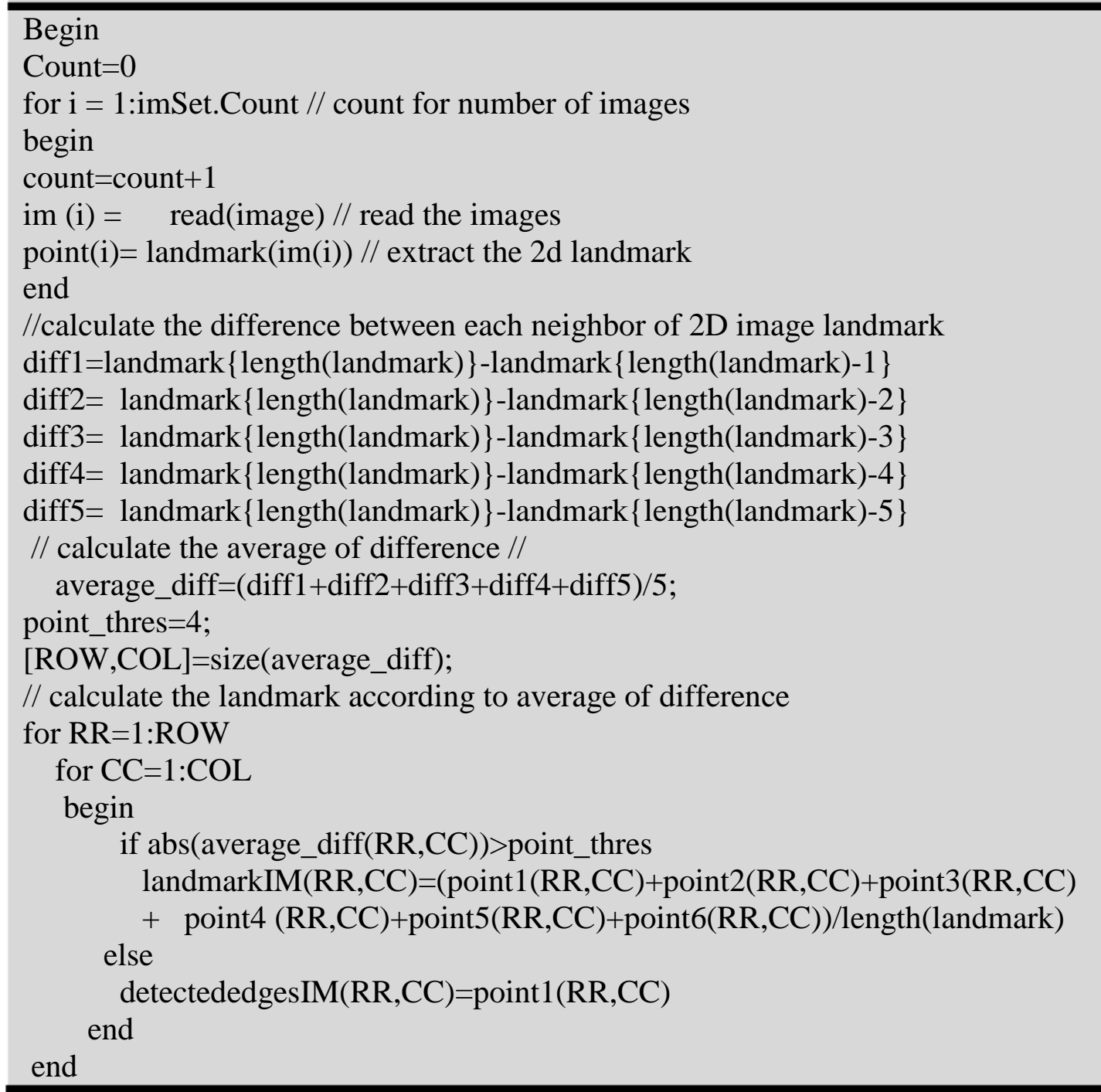




\section{Algorithm(3): Extraction of Pose and Shape Parameters}

Input: landmark of 2D image, (parameter of 3DMM),xyzpoints

Output: pose parameters (R, T) and shape parameter ( FACE.vertices)

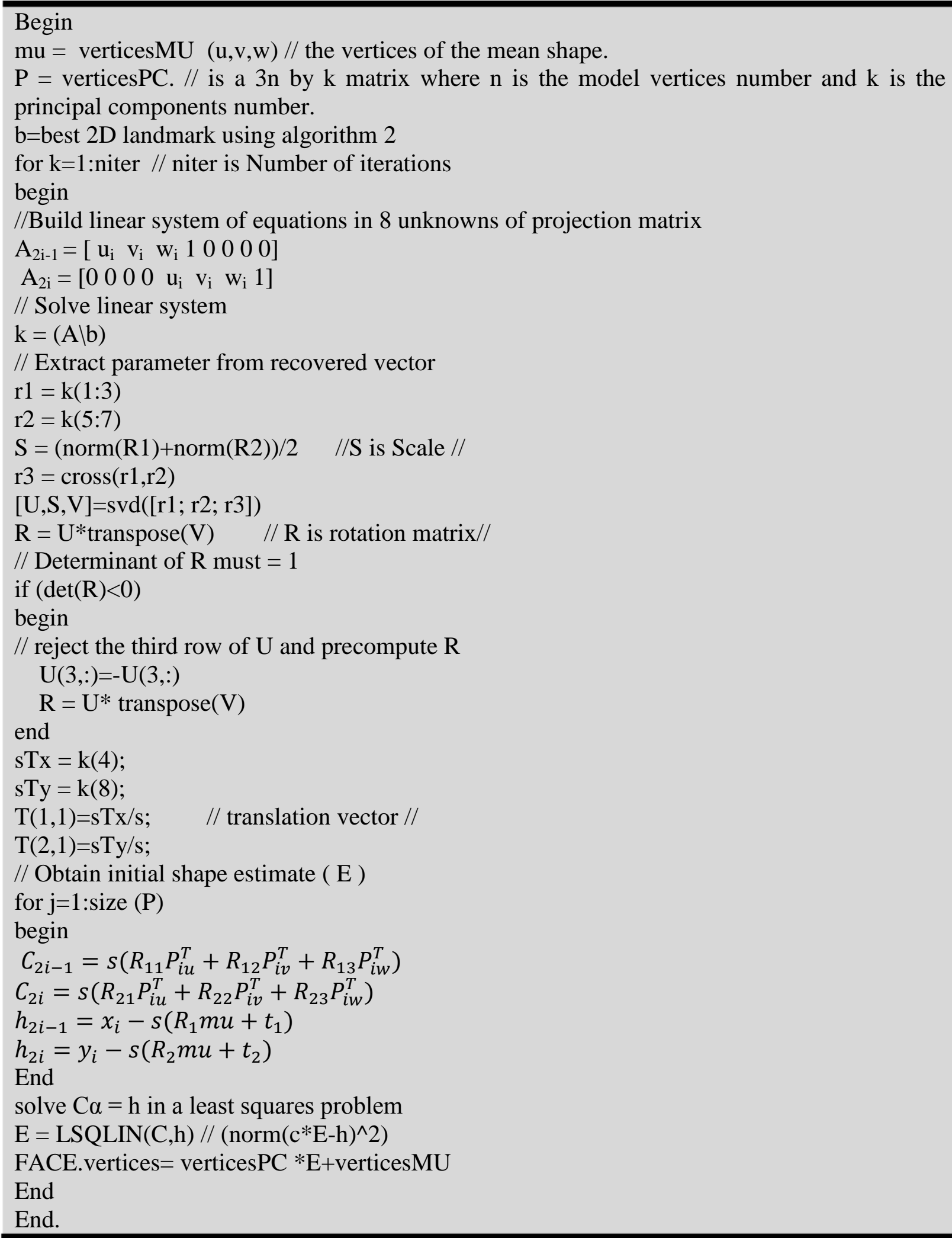


* Perform 3DMM fitting using 2D

landmark and edges as shown in subsection 3.2. In this stage get the final shape parameter to the final 3D model.
Perform an iterative procedure to change the shape parameter according to $2 \mathrm{D}$ edge until get the accurate $3 \mathrm{D}$ model as shown in algorithm(4).

\section{Algorithm(4): 3DMM Fitting using 2D Landmark and Edges}

Input: edge of 2D image, (parameter of 3DMM)

Output: finial shape parameter

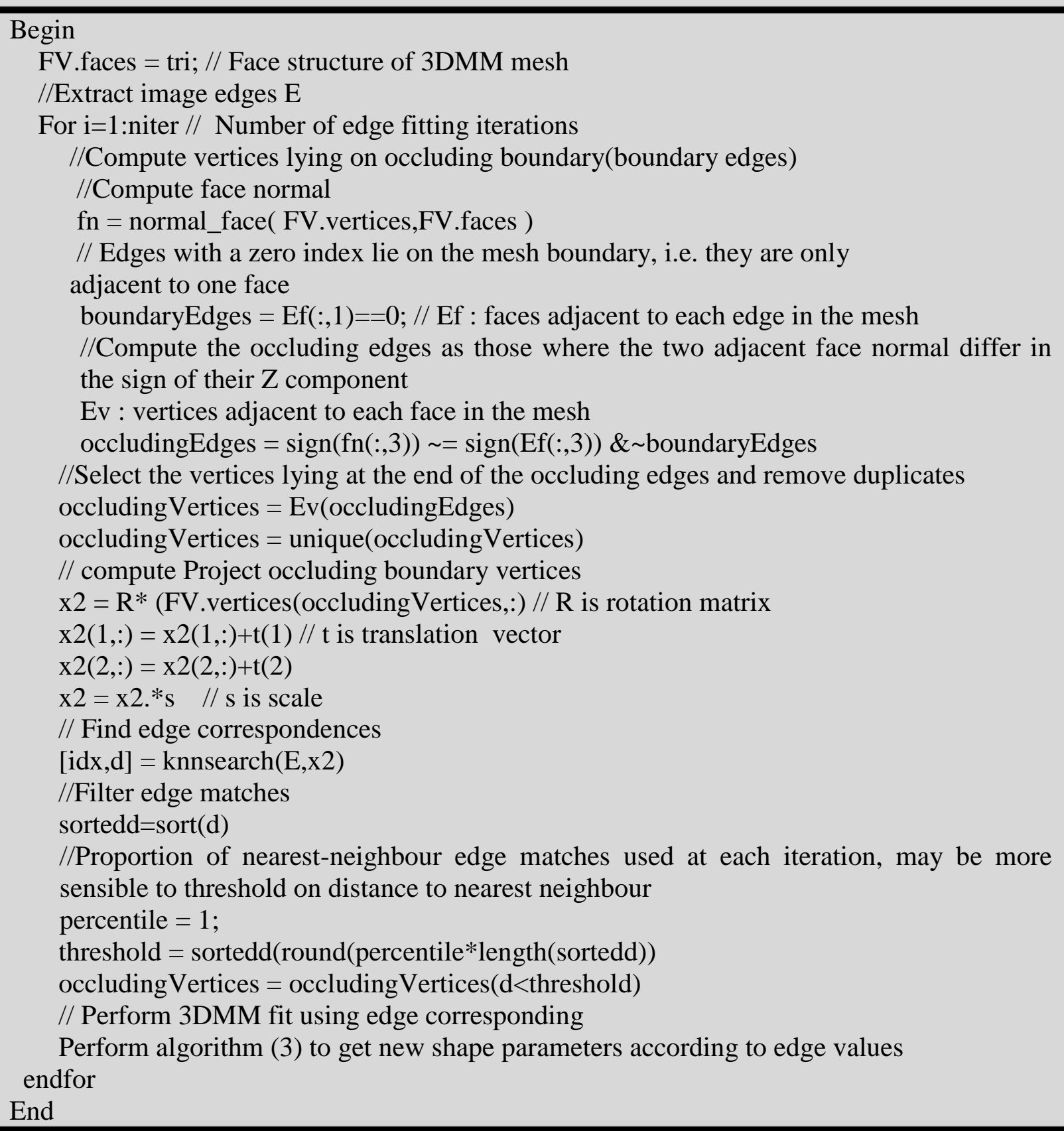


Shaimaa .H / Najlaa .A

\section{Results and Discussion:}

In this work, available comprehensive and popular 2D image database Psychological Image Collection at Stirling (PICS) is used. This dataset contains 687 Colour faces, Between 1 and 18 images of 90 individuals. Its variations in pose, viewpoint, and expression.
The database's resolution is varied from $336 \times 480$ to $624 \times 544$. Fig. 4 shows the unconstrained image sequence (different in poses) which represent the input for the system.

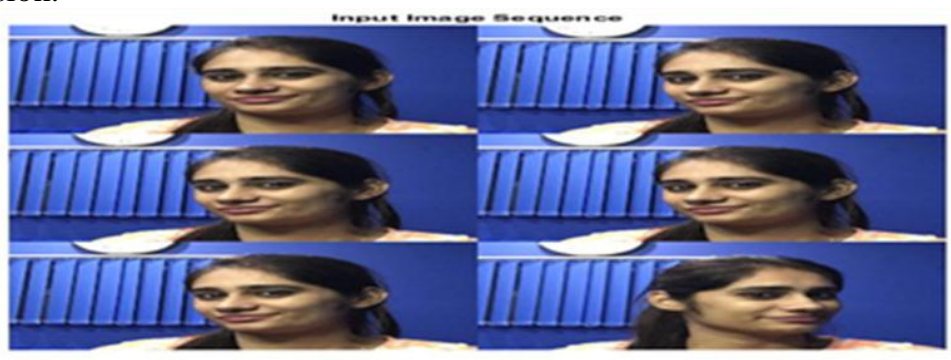

Figure 4: Input image sequence.

Fig.5 (a) represents the generic model, then deforms this model by fitting with the $2 \mathrm{D}$ image. First the generic model deforms according to only 2D feature (landmark) as shown in figure 5 (b), then begins in iterative procedure to deform

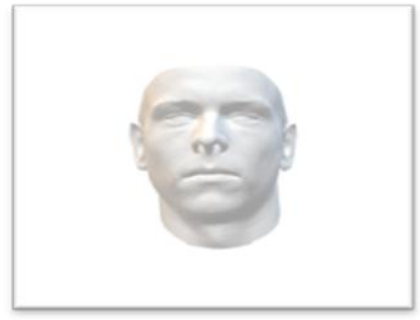

(a) the 3d model with 2D landmark and edges by finding nearest neighbor between model edges and image edges to get the finial 3D model which match with the feature in $2 \mathrm{D}$ image as shown in figure 6

Figure 5 (a): the initial 3D model. (b): the initial deform 3D model

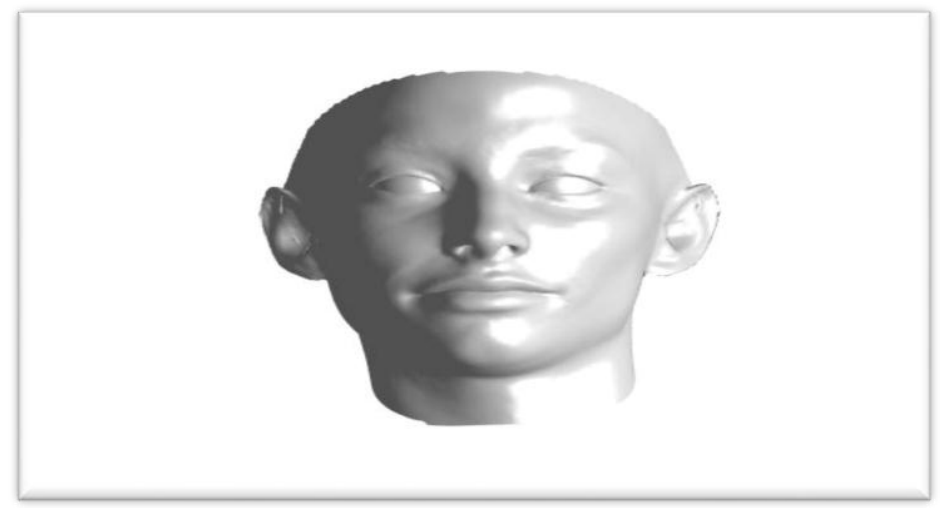

Figure 6 : finial 3D model. 
Shaimaa .H / Najlaa .A

As shown in figure 6 the proposed system can deform the initial 3D model (figure ${ }^{\circ}$ a) in order to be corresponding with $2 \mathrm{D}$ feature, and can change the feature from male in initial model to female feature (figure 6).

\subsection{Comparison between traditional fitting procedure and the proposed fitting method:}

In this subsection shows the performance the proposed fitting procedure and compare with the classical method as shown in table 1.

Table 1: Comparison between traditional fitting procedure and the proposed fitting method

\begin{tabular}{|l|l|l|}
\hline $\begin{array}{l}\text { Deform 3D model according to traditional } \\
\text { method }\end{array}$ & Deform 3D model according to the proposed method \\
\hline & & \\
\hline
\end{tabular}

As shown in above table, the proposed system gives 3D model more accurate and smooth from the traditional method.

\section{Conclusions}

In this paper new 3D face reconstruction method is designed by improvement fitting procedure to get accurate $3 \mathrm{D}$ model by taking best $2 \mathrm{D}$ landmark of all images rather than taking only first 2D image landmark (traditional method). this modify is shown by comparing the proposed system with the traditional method. The main conclusions that are achieved from the proposed system are:

1. The proposed system can be applied to any pose of the input image.

2. The proposed 3D reconstruction has achieved good accuracy according to deform general 3D model to correspond the 2D feature.
3. the proposed system can overcome extremely challenge which is deforming the $3 \mathrm{D}$ default model to make it compatible with the 2D feature especially female feature.

\section{References}

[1]G. Stylianou and A. Lanitis, "Image Based 3D Face Reconstruction: a Survey", International Journal of Image and Graphics, vol. 9, no. 2, pp. 217-250, 2009.

[2] A. Jourabloo, "A Survey of Different 3D Face Reconstruction Methods", Technical Report, 2016

[3]J. Roth, Y. Tong, and X. Liu, "Unconstrained 3D Face Reconstruction", IEEE Xplor , 2015.

[4]M. Dikmen, "3D Face Reconstruction Using Stereo Vision" M.Sc thesis, Middle East Technical University, 2006. 
[5] J. Roth, Y. Tong, and X. Liu, "Adaptive 3D Face Reconstruction from Unconstrained Photo Collections", IEEE Transactions on Pattern Analysis and Machine Intelligence, 2016.

[6] M. Siudak and P. Rokita, "A Survey of Passive 3D Reconstruction Methods on the Basis of More Than One Image" Machine Graphics \& Vision, vol. 23, no. 3, pp. 57-117, 2014.

[7] G. Hu, "Face Analysis using 3D Morphable Models", Ph.D. Thesis, University of Surrey, 2015.

[8] S. Se and N. Pears, "3D Imaging, Analysis and Applications", Book Published in the Springer, 2012.

[9] H. Kjer and J. Wilm, "Evaluation of surface registration algorithms for PET motion correction" Thesis, Technical University of Denmark , 2010.

[10] C. Soler, " Non-rigid registration of 3D

face scans Carlota Soler Arsasanz

Bachelor", Master Thesis, 2015.
[11] M. Piotraschke and V. Blanz," Automated 3D Face Reconstruction from Multiple Images using Quality Measures", IEEE Explore,pp. 3418-3427,2016.

[12] A. Bas, W. Smith, T. Bolkart, and S. Wuhrer, "Fitting a 3D morphable model to edges: A comparison between hard and soft correspondences" Lecture Notes in Computer Science, pp. 377-391, 2017.

[13] Z. Xiangxin and R. Deva, "Face detection, pose estimation, and landmark estimation in the wild", Dept. of Computer Science, University of California, Irvine, CVPR, PP. 2879-2886, 2012.

\section{إعادة بناء الوجوه الثلاثية الابعاد باستخدام تقنية مطابقة نموذج مورفبل الثُلاثي الابعاد}
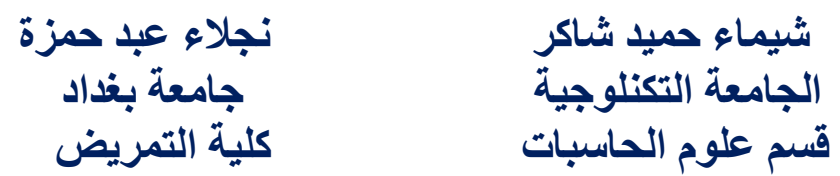

(المستخلص : (1)

في هذا البحث، تم در اسة إعادة بناء الوجوه في شكل نموذج ثناثي الأبعاد للصور غير المقيدة (ذات أوضاع مختلفة). وقد

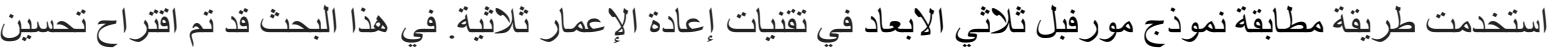

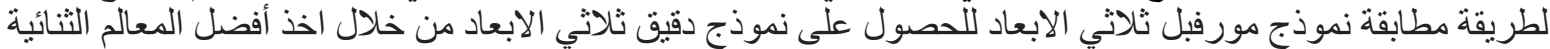

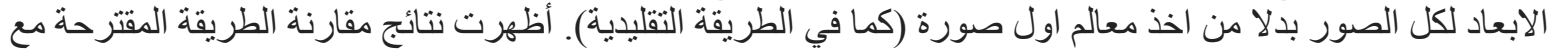
الطريقة التقليدية نتائج مشجعة من ناحية وقت الإن التنفيذ وجودة إعادة البناء. 